\title{
Bitcoin over Tor isn't a good idea
}

\author{
Alex Biryukov \\ University of Luxembourg \\ Email: alex.biryukov@uni.lu
}

\author{
Ivan Pustogarov \\ University of Luxembourg \\ Email: ivan.pustogarov@uni.lu
}

\begin{abstract}
Bitcoin is a decentralized P2P digital currency in which coins are generated by a distributed set of miners and transactions are broadcasted via a peer-to-peer network. While Bitcoin provides some level of anonymity (or rather pseudonymity) by encouraging the users to have any number of random-looking Bitcoin addresses, recent research shows that this level of anonymity is rather low. This encourages users to connect to the Bitcoin network through anonymizers like Tor and motivates development of default Tor functionality for popular mobile SPV clients. In this paper we show that combining Tor and Bitcoin creates a new attack vector. A low-resource attacker can gain full control of information flows between all users who chose to use Bitcoin over Tor. In particular the attacker can link together user's transactions regardless of pseudonyms used, control which Bitcoin blocks and transactions are relayed to user and can delay or discard user's transactions and blocks. Moreover, we show how an attacker can fingerprint users and then recognize them and learn their IP addresses when they decide to connect to the Bitcoin network directly.
\end{abstract}

\section{INTRODUCTION}

Bitcoin is a decentralized virtual currency and a P2P payment system in which coins are generated by miners and double spending is prevented by that each peer keeps a local copy of the constantly growing public ledger of all the previous transactions. Though the original Bitcoin paper states that privacy in such a system may still be maintained, the recent findings disprove this. Anonymity and privacy of the plain Bitcoin protocol is also not claimed by the Bitcoin developers.

There are two independent problems: a) ability of the attacker to link transactions to the IP address of the user [2], [15], [14] by studying connectivity and traffic of the peers and b) linkability of the user's pseudonyms and transactions in the public ledger achieved via transaction flow analysis [20], [17]. At the same time as Bitcoin increases its user base and moves from mining and hoarding to the actual use as a currency and payment protocol in various on-line applications there is a growing demand in more privacy among the Bitcoin users. While one could use a Bitcoin mixing service $^{1}$ to break connections in the transaction graph, IP address leakage is still possible. Bitcoin developers recommend to use third party anonymization tools like Tor or VPNs to solve this problem.

Some alternative currencies like Anoncoin, BitTor, Torcoin, Stealthcoin, and others offer native support for Tor. There are also several other use cases for Tor in the Bitcoin ecosystem. For mobile payments it is of interest to use so called SPV (simple payment verification) clients which cannot afford to hold the full 20 Gbyte blockchain ledger. Such feature was

${ }^{1}$ This is always a matter of trust of the service operator. already foreseen in the original Bitcoin whitepaper, see Section 8 of [18]. Since such popular clients [5], [11] (around 1 Million expected userbase [13]) are vulnerable to spoofing attacks which may result in double-spending (see appendix B), the current trend is to bundle them with Tor by default to avoid spoofing ${ }^{2}$ and man-in-the-middle attacks. Tor can also be a solution for services and online shops that want to prevent DoS attacks against their public IP. Finally Tor is seen as a countermeasure if Internet neutrality towards Bitcoin will start to erode [7].

Tor is not a panacea however and not all applications are anonymized equally well when combined with Tor. The biggest effort has been made so far on improving protection of the HTTP $(\mathrm{S})$ protocol on top of Tor. Other protocols are not researched that well. There were several documented cases when application level leaked crucial user-identifying information [16], [23]. Moreover, there is only limited number of applications which are studied well enough to be considered safe to use with Tor [25].

This paper contains two main contributions: first we show that using Bitcoin through Tor not only provides limited level of anonymity but also exposes the user to man-in-the-middle attacks in which an attacker controls which Bitcoin blocks and transactions the user is aware of. The estimated cost of the attack is below 2500 USD per month.

The second main contribution is a fingerprinting technique for Bitcoin users by setting an "address cookie" on user's computer. This can be used to correlate the same user across different sessions, even if he uses Tor, hidden-services or multiple proxies. If the user later decides to connect to the Bitcoin network directly the cookie would be still present and would reveal his IP address. A small set of Sybil nodes (about a 100 attacker's nodes) is sufficient to keep the cookies fresh on all the Bitcoin peers (including clients behind NATs).

The man-in-the-middle attack exploits a Bitcoin built-in reputation based DoS protection and the attacker is able to force specific Bitcoin peers to ban Tor Exit nodes of her choice. Combining it with some peculiarities of how Tor handles data streams a stealthy and low-resource attacker with just 1-3\% of overall Tor Exit bandwidth capacity and 1000-1500 cheap lightweight Bitcoin peers (for example, a small Botnet) can force all Bitcoin over Tor traffic to go either through her Exit nodes or through her peers. This opens numerous attack vectors. First, it simplifies traffic correlation attack since the attacker controls one end of the communication. Second, the attacker can glue together different Bitcoin addresses (pseudonyms) of the same user. Third, it opens possibilities of

\footnotetext{
${ }^{2}$ E.g. when connecting through a public Wi-Fi.
} 
double spending attacks for mobile SPV clients, those which it was supposed to protect from such attacks. Moreover in collusion with a powerful miner double-spending becomes possible and a totally virtual Bitcoin reality may be created for such users (at least for a brief period of time).

The rest of the paper is organized as follows. In Section II, we provide information on Bitcoin and Tor internals required for understanding the attacks. In Section III we describe how an attacker can get in the middle between Bitcoin clients and Bitcoin network, effectively isolating the clients from the rest of the Bitcoin P2P network. We also show that Bitcoin peers available as Tor hidden services may not solve the problem. In Section IV, we show how a user can be fingerprinted and his activity linked across different sessions. Section V describes how the attacker can increase the probability that a user connecting to the Bitcoin network directly (i.e. without using Tor) will choose her peers. In Section VI, we analyze the man-in-the-middle attack and estimate connection delays experienced by the user and check for which malicious Exit bandwidth and number of malicious peers the attack becomes unnoticeable to the user. Section VII calculates the costs of the attack. In Section VIII, we describe several possible countermeasures.

Ethical considerations. We reported the attacks described in this paper to the Bitcoin developers. In addition we submitted a patch which fixes the "address cookie" attack. Though we carried out some experiments using our own relays in the real Bitcoin and Tor networks we believe that no users were harmed since the relays were limited in bandwidth and were running only for a short time. Address cookie fingerprinting was tested only on our own clients/transactions.

\section{BACKGROUND}

In this section we provide details of the inner working of Tor and Bitcoin protocols. Many of these details were obtained by an analysis of the corresponding source $\operatorname{code}^{3}$. This is especially true for Bitcoin for which there exists no official documentation except for the original white paper [18] and Bitcoin Wiki [4].

\section{A. Bitcoin}

Bitcoin is a decentralized virtual currency and a payment system based on cryptography and a peer-to-peer network. Its main components are transactions and blocks. Blocks are created by Bitcoin miners by solving cryptographic puzzles of controlled hardness (called proofs of work). The proof of work consists of finding a cryptographic hash value for a block of transactions which starts with a certain number of leading zero bits (32 when Bitcoin was first proposed, 67 zero bits at present). With each solved block a miner creates and earns 25 new Bitcoins. Hash of the previous block is included into the new block, which results in a chain of blocks or blockchain. The difficulty of the cryptographic puzzles is adjusted automatically by the network so that the network generates one block every 10 minutes on the average. Payers and payees of the system are identified by Bitcoin addresses which are base 58 -encoded hashes of their public keys. Money transfers from one Bitcoin address to another are done by

${ }^{3}$ Bitcoin version 0.9.2 and Tor version 0.2.4.23 creating a signed transaction and broadcasting it to the P2P network. Transactions are included into blocks by miners; once a transaction is buried under a sufficient number of blocks, it becomes computationally impractical to double spend coins in this transaction.

Bitcoin is a peer-to-peer system where each peer is supposed to keep its copy of the blockchain, which plays a role of a public ledger. Whenever a block or a transaction is generated by a peer, it is broadcasted to other peers in the network. Upon receipt and verification of the block's proof of work the peer updates his copy of the blockchain. Bitcoin software does not explicitly divide its functionality between clients and servers, however Bitcoin peers can be grouped into those which accept incoming connections (servers) and those which don't (clients), i.e. peers behind network address translation (NAT) or firewalls. Bitcoin users connecting to the Bitcoin network through Tor or VPN obviously also do not accept incoming connections.

At the time of writing there are about 7,000 reachable Bitcoin servers [6] and the number of clients is estimated to be an order of magnitude larger [8]. By default Bitcoin peers (both clients and servers) try to maintain 8 outgoing connections to other peers in the network. If any of the 8 outgoing connections drop, a Bitcoin peer tries to replace them with new connections. Using our terminology, a Bitcoin client can only establish a connection to a Bitcoin server. We call servers to which a client established connections entry nodes of this client. By default a server can accept up to 117 incoming connections. If this limit is reached all new connections are dropped.

1) Bitcoin anti-DoS protection: As an anti-DoS protection, Bitcoin peers implement a reputation-based protocol with each node keeping a penalty score for every other Bitcoin peer (identified by its IP address). Whenever a malformed message is sent to the node, the latter increases the penalty score (different messages incur different penalties) of the sender and bans the "misbehaving" IP address for 24 hours when the penalty reaches the value of 100 .

2) Bitcoin peers as Tor hidden services: Tor hidden services (see section II-B3) are service-agnostic in the sense that any TCP-based service can be made available as a Tor hidden service. This is used by Bitcoin which recognizes three types of addresses: IPv4, IPv6, and OnionCat [19]. Onioncat address format is a way to represent an onion address as an IPv6 address: the first 6 bytes of an OnionCat address are fixed and set to FD87:D87E:EB43 and the other 10 bytes are the hex version of the onion address (i.e. base 32 decoded onion address after removing the ".onion" part).

3) Bitcoin peer discovery and bootstrapping: Bitcoin implements several mechanisms for peer discovery and bootstrapping. First, each Bitcoin peer keeps a database of IP addresses of peers previously seen in the network. This database survives between Bitcoin client restarts. This is done by dumping the database to the hard drive every 15 minutes and on exit (as we will see later this facilitates setting a cookie on the user's computer). Bitcoin peers periodically broadcast their addresses in the network. In addition peers can request addresses from each other using GETADDR messages and advertise addresses using ADDR messages. 
If Tor is not used, when a Bitcoin clients starts, it first tries to populate its address database by resolving 6 hard-coded hostnames ${ }^{4}$. If Tor is used, Bitcoin does not explicitly ask Tor to resolve $\mathrm{e}^{5}$ them but rather asks it to establish connections to these hostnames.

If Tor is not used, the addresses for outgoing connections are taken from the addresses database only. In case Tor is used, every second connection is established to a DNS hostname. These DNS hostnames are called "oneshots" and once the client establishes a connection to such a hostname it requests a bunch of addresses from it and then disconnects and never tries to connect to it again. As a fallback if no addresses can be found at all, after 60 seconds of running the Bitcoin client uses a list of 600 hard-coded IP addresses.

Bitcoin nodes recognize three types of addresses: IPv4, IPv6, and OnionCat [19]. For each type of addresses the peer maintains a state variable indicating if the Bitcoin node is capable of using such address type. These state variables become important when using Tor: the only address type which is accepted from other peers is OnionCat type. Curiously, this results in that all IPv4 and IPv6 addresses obtained from oneshots are dropped and the client uses its original database. The opposite case also holds: if Tor is not used, onion addresses are not stored in the address database.

Finally each address is accompanied by a timestamp which determines its freshness.

4) Choosing outgoing connections: For each address in the address database, a Bitcoin peer maintains statistics which among other things includes when the address was last seen in the network, if a connection to this address was ever established before, and the timestamp of such connection. All addresses in the database are distributed between so called buckets. There are 256 buckets for "new" addresses (addresses to which the Bitcoin client has never established a connection) and 64 for "tried" addresses (addresses to which there was at least one successful connection). Each bucket can have at most 64 entries (which means that there can be at most 20480 addresses in the database). When a peer establishes outgoing connections, it chooses an address from "tried" buckets with probability $p=0.9-0.1 n$, where $n$ is the number of already established outgoing connections. If an address is advertised frequently enough it can be put into up to 4 different "new" buckets. This obviously increases its chances to be selected by a user and to be transferred to a "tried" bucket.

\section{B. Tor}

Tor is the most popular low-latency anonymity network which at the time of this writing comprised 6000-7000 routers with an estimated number of daily users exceeding 500,000 (not counting the botnet-infected nodes). Tor is based on ideas of onion routing and telescoping path-building design. When a user wants to connect to an Internet server while keeping his IP address in secret from the server he chooses a path consisting of three Tor relays (called Guard, Middle and Exit), builds a

\footnotetext{
${ }^{4}$ At the time of this writing one of these hostnames constantly failed to resolve into any IP address.

${ }^{5}$ When applications communicate with Tor they can either ask Tor to establish a connection to a hostname by sending a CONNECT command or to resolve a hostname by sending a RESOLVE command.
}

circuit and negotiates symmetric keys with each one of them using the telescoping technique. Before sending a message to the server, the user encrypts it using the negotiated keys. While the message travels along the circuit, each relay strips off its layer of encryption. In this way the message arrives at the final destination in its original form and each party knows only the previous and the next hop.

Tor tries hard to achieve low traffic latency to provide a good user experience, thus sacrificing some anonymity for performance. To keep latency low and network throughput high, Tor relays do not delay incoming messages and do not use padding. This makes Tor susceptible to traffic confirmation attacks: if an attacker is able to sniff both ends of a communication, she is able to confirm that the user communicated with the server. If the first hop of a circuit is chosen at random then the probability that a malicious node will be chosen as the first hop (and thus will know the IP address of the user) converges to one with the number of circuits. Due to this, each user has a set of three ${ }^{6}$ Guard nodes. When a user builds a circuit the first hop is chosen from the set of Guard nodes.

The list of all Tor relays is assembled and distributed in the so called consensus document by nine trusted Tor authorities. For the purpose of traffic balancing the bandwidth of each relay is measured and reported. A user chooses relays for his circuits with probability proportional to the relays' weights listed in the consensus ${ }^{7}$. Each relay in the consensus is identified by his fingerprint (or ID) which is the SHA-1 hash of its public key.

1) Tor stream timeout policy: Tor provides SOCKS interface for applications willing to connect to the Internet anonymously. Each connection to the SOCKS port by an application is called a stream. For each new stream Tor tries to attach it either to an existing circuit or to a newly built one. It then sends a BEGIN cell down the circuit to the corresponding Exit node asking it to establish a connection to the server requested by the application. In order to improve user's quality of service, if Tor does not receive a reply from the Exit node within 10 or 15 seconds $^{8}$, it drops the circuit and tries another one. If none of the circuits worked for the stream during 2 minutes, Tor gives up on it and sends a SOCKS general failure error message.

2) Tor Exit policy: In order to access a Web resource anonymously through a Tor circuit, the Exit relay (the final relay in the circuit) should allow establishing connections outside the Tor network. This makes Exit relay operators open to numerous abuses. In order to make their life easier, Tor allows them to specify an Exit Policy: a list of IP addresses and ports to which the Exit node is willing to establish connections and which destination are prohibited. When a client establishes a circuit, he chooses only those Exit nodes which allow connections to the corresponding IP addresses ${ }^{9}$ and port ranges.

\footnotetext{
${ }^{6}$ Will be reduced down to one Guard per user in the next Tor update [24].

${ }^{7}$ This is a simplification of the real selection procedure in which additional weights are assigned to a relay based on its position in the circuit and its flags.

${ }^{8}$ Tor waits for 10 seconds for the first two attempt and 15 seconds for the subsequent attempts.

${ }^{9}$ Note that usually at the time the path is selected, only the domain name is known.
} 
3) Tor Hidden Services: Tor is mostly known for its ability to provide anonymity for clients accessing Internet services. Tor Hidden Services are another feature of Tor which enables responder anonymity: a service can be contacted by clients without revealing its physical location. In order to achieve this a client and the hidden service choose at random and connect to a Tor relay (rendezvous point) and forward all the data through it. In more detail:

1) The hidden service generates a public key and chooses at random a small number of Tor relays (typically three) which become its introduction points. The service maintains permanent connection to these relays.

2) It then generates an HS descriptor which contains the public key and the list of introduction points, and

3) Publishes it at 6 different Tor relays having HSDir flag ${ }^{10}$. These are called responsible $H S$ directories. The choice of responsible HS directories is deterministic and depends on the hash of the hidden service's public key and current day.

4) Introduction points are instructed by the hidden service to forward connection requests from clients. The base 32 encoding of the hash of the hidden service's public key (onion address) is then communicated to clients by conventional means (blog post, e-mail, etc.).

When a client decides to connect to the hidden service, he:

1) Determines the list of the responsible HS directories using the onion address and downloads the HS descriptor.

2) Chooses a rendezvous point at random.

3) Communicates the ID of the rendezvous point to the hidden service's introduction points which then forward it to the hidden service.

When the hidden service receives the ID of the rendezvous point, it establishes a connection to it and the data transfer between the service and the client can start. All communications between the client and the rendezvous point, between the service and the rendezvous point and between the service and the introduction points are established over three-hop circuits. This hides the location of the hidden service and its clients both from each other and from external observer.

The hidden service or a client can determine the fingerprints of the responsible directories as follows. They first take all Tor relays which have HSDir flag in the consensus and sort their fingerprints in lexicographical order. Second, they compute the descriptor ID's of the hidden service which is the SHA-1 hash of a value composed of the following items ${ }^{11}$ : public key of the hidden service, current day, and replica (which can be 0 or 1). The exact expression for the ID is of little importance here, the only important things are a) the ID changes every 24 hours, b) there are two replicas of the ID.

\footnotetext{
${ }^{10}$ HSDir flag is assigned by Tor authorities to relays which wish to be a part of a distributed database to store descriptors of Tor hidden services. A relay should be running for at least 25 hours to get this flag.

${ }^{11} \mathrm{~A}$ hidden service may also decide to use a secret key (somewhat misleadingly called descriptor-cookie), but for hidden services which are meant to be accessed by everybody it is not relevant.
}

Third they find the place in the sorted list of the fingerprints for the computed ID and take the next three relays' fingerprints (thus having 6 fingerprints it total since there are two replicas).

\section{GETTING IN THE MIDDLE}

By exploiting Bitcoin's anti-DoS protection a low-resource attacker can force users which decide to connect to the Bitcoin network through Tor to connect exclusively through her Tor Exit nodes or to her Bitcoin peers, totally isolating the client from the rest of the Bitcoin P2P network. This means that combining Tor with Bitcoin may have serious security implications for the users: 1) they are exposed to attacks in which an attacker controls which Bitcoin blocks and transactions the users are aware of; 2) they do not get the expected level of anonymity.

The main building blocks of the attack are: Bitcoin's reputation-based anti-Dos protection, Tor's stream management policy, the fact that connections between Bitcoin peers are not authenticated. Authors in [2] exploited the Bitcoin's reputation-based DoS protection to force all Bitcoin servers to ban all Tor Exit nodes. In this section we exploit the DoS protection, however we noticed that instead of just baning Bitcoin clients from using Tor the attacker might achieve much smarter results. The attack consists of four steps:

- Inject a number of Bitcoin peers to the network. Note that though Bitcoin allows only one peer per IP address, it does not require high bandwidth. IP addresses can be obtained relatively cheaply and on per-hour basis.

- Periodically advertise the newly injected peers in the network so that they are included into the maximum possible number of buckets at the client side.

- Inject some number of medium-bandwidth Tor Exit relays. Even a small fraction of the Exit bandwidth would be enough for the attacker as will be shown later.

- Make non-attacker's Bitcoin peers ban non-attacker's Tor Exit nodes.

We now explain each step of the attack in more detail. See section VI for attack parameter estimation.

\section{A. Injecting Bitcoin peers}

This step is rather straightforward. In order to comply with Bitcoin's limitation "one peer per IP address", the attacker should obtain a large number of IP addresses. The easiest way would be to rent IP addresses on per hour basis. The market value is 1 cents per hour per IP address [21]. The important note is that the obtained IP addresses will not be involved in any abusive activity (like sending spam or DoS attacks) which makes this part of the attack undetectable.

\section{B. Advertising malicious peers}

The attacker is interested in that her Bitcoin peers are chosen by Bitcoin clients as frequently as possible. In order to increase by factor four the chances for her peers to be included into "tried" buckets, the attacker should advertise the addresses 
of her peers as frequently as possible. This mechanism would allow the attacker to inject less malicious peers. Note also that address advertisement is not logged by default and thus requires special monitoring to be noticed.

\section{Injecting Tor Exit nodes}

During this step the attacker runs a number of Exit Tor nodes. In order to get Exit flag from the Tor authorities, an attacker's Exit node should allow outgoing connections to any two ports out of ports 80, 443, or 6667. Such an open Exit policy might not be what a stealthy attacker wants. Fortunately for the attacker she can provide incorrect information about her exit policy in her descriptor and thus have Exit flag while in reality providing access to port 8333 only. The attacker can do even better, and dynamically change the exit policy of her relays so that only connections to specific Bitcoin peers are allowed. We implemented this part of the attack: while the Tor consensus indicated that our relays allowed exiting on ports 80 , 443, and 8333 for any IP address, the real exit policy of our relays was accepting port 8333 for a couple of IP addresses ${ }^{12}$.

\section{Banning Tor Exit nodes}

In this phase, the attacker exploits the built-in Bitcoin antiDoS protection. The attacker chooses a non-attacker's Bitcoin peer and a non-attacker's Tor Exit, builds a circuit through this Exit node and sends a malformed message to the chosen Bitcoin peer (e.g. a malformed coinbase transaction which is 60 bytes in size and which causes the immediate ban for 24 hours). As soon as the Bitcoin peer receives such message it analyses the sender's IP address which obviously belongs to the Tor Exit node chosen by the attacker. The Bitcoin peer then marks this IP address as misbehaving for 24 hours. If a legitimate client then tries to connect to the same Bitcoin peer over the banned Exit node, his connection will be rejected. The attacker repeats this step for all non-attacker's Bitcoin peers and each non-attacker's Tor Exit node. This results in that a legitimate Bitcoin user is only able to connect to Bitcoin over Tor if he chooses either one of the attacker's peers or establishes a circuit through an attacker's Exit node. We validated this part of the attack by forcing about 7500 running Bitcoin peers to ban our Exit node. To do this we implemented a rudimentary Bitcoin client which is capable of sending different custom-built Bitcoin messages.

\section{E. Defeating onion peers}

Bitcoin peers can be made reachable as Tor hidden services. Banning Tor Exit nodes will obviously not prevent Bitcoin clients from connecting to such peers. Nonetheless our observations show that this case can also be defeated by the attacker.

First the current design of Tor Hidden Services allows a low-resource attacker to DoS a hidden service of her choice [3] (this technique is called black-holing of hidden services). Before a client can contact a hidden service he needs to download the corresponding descriptor from one of the six responsible hidden service directories. These directories are chosen from the whole set of Tor relays in a deterministic way based on the onion address and current day (see section II-B3).

\footnotetext{
${ }^{12}$ We also allowed exiting to IP addresses used by Tor bandwidth scanners.
}

The attacker needs to inject six malicious relays that would become responsible directories. In other words she needs to find the right public keys with fingerprints which would be in-between the descriptor IDs of the hidden service and the fingerprint of the currently first responsible hidden service directory. Authors in [3] show that computationally it is easy to do. It can become a problem though for a large number of hidden services: for each hidden service the attacker needs to run at least 6 Tor relays for at least 25 hours, 2 relays per IP address.

Fortunately for the attacker the fraction of Bitcoin peers available as Tor hidden services is quite small. During August 2014 we queried address databases of reachable Bitcoin peers [6] and among 1,153,586 unique addresses (port numbers were ignored), only 228 were OnionCat addresses and only 39 of them were actually online; in November 2014 we repeated the experiment and among 737,314 unique addresses 252 were OnionCat addresses and 46 were online (see Appendix A for the two lists of these Bitcoin onion addresses). This results in (1) a very small probability for a client to choose a peer available as a hidden service; (2) this makes black-holing of existing Bitcoin hidden services practical.

Second, the attacker can at almost no cost inject a large number of Bitcoin peers available as Tor hidden services. It requires running only one bitcoind instance and binding it with as many onion addresses as needed. Thus users will more likely connect to attacker controlled "onion" peers.

Third, as was described in section II-A3, when running Bitcoin without Tor, onion addresses received from peers are silently dropped. Thus one can only obtain OnionCat addresses by either connecting to an IPv4- or IPv6-reachable peers through a proxy ${ }^{13}$ or by specifying an onion address in the command line.

\section{F. Attack vectors}

The technique described in this section allows an attacker to direct all Bitcoin-over-Tor traffic through servers under her control. This creates several attack vectors which we will briefly describe in this subsection.

Traffic confirmation attack. First, it becomes much cheaper to mount a successful traffic confirmation attack. In traffic confirmation attacks, the attacker controls a fraction of Guard and Exit nodes. The attacker sees that one of her exit nodes is requested to access a particular (e.g. censored) web-site and the attacker is interested in finding out the user who made this request. The attacker sends a traffic signature down the corresponding circuit. If the attacker was lucky and the user chose one of her Guard nodes, the attacker will see the traffic signature going through this Guard to the target user. This reveals the user's IP address.

The success probability of the attack is computed as the product of two factors: the probability for the user to choose an attacker's Guard and the probability for the user to choose an attacker's Exit. Since now all exit Bitcoin-over-Tor traffic goes through the attacker, the second factor becomes 1 .

Revealing Guard nodes. In case the attacker does not control the user's Guard node, he may try to find this Guard. We

\footnotetext{
${ }^{13}$ Not necessarily Tor.
} 
assume that the attacker controls a fraction of middle nodes. As before the attacker would send a traffic signature down the circuit and if none of the attacker's middle nodes detects this signature, the attacker drops the circuit. This will force the user to build another circuit. After some number of circuit tries, one of the attacker's middle nodes will finally be chosen. This middle node will know the user's Guard node. The reidentification of the user between different circuits is possible e.g. using the fingerprinting technique from section IV.

Revealing the guards does not immediately allow an attacker to reveal the location of the user but gives her the next point of attack. Given that guard nodes are valid for more than a month, this may be sufficient to mount a legal attack to recover traffic meta data for the guard node, depending on the jurisdiction the guard node is located in.

Linking different bitcoin addresses. Even without knowing the user's IP, the attacker can link together user's transactions regardless of pseudonyms used.

Possibility of double spending. Finally, after successfully mounting the attack described in this section the attacker controls the connectivity to the Bitcoin network for users which chose to use Tor. This increases the success rate of double-spend attacks described in Appendix B.

In addition the attacker can defer transactions and blocks and send dead forks. In collusion with a powerful mining pool (for example $10-20 \%$ of total Bitcoin mining capacity) the attacker can create fake blocks. This enables additional possibilities for double spending, however to make this relevant the amount should exceed what such miner would be able to mine in the real Bitcoin network. Also complete alternative Bitcoin reality for all the users who access Bitcoin solely through Tor is possible. This however would come at a cost of 5-10 times slower confirmations, which after some time can be detected by the wallet software.

\section{USER FINGERPRINTING}

In this section we describe a technique which can be used to fingerprint Bitcoin users by setting an "address cookie" on their computers. A cookie can be set and checked even when the user connects to the Bitcoin network through Tor or through a chain of proxies. It can be used to correlate different transactions of the same user even across different sessions (i.e. after his computer was rebooted). If the user decides later to send a non-sensitive transaction without Tor, his fingerprint can be correlated to his IP address, thus deanonymizing all his transactions sent previously through Tor. The fingerprinting technique is based on the Bitcoin's peer discovery mechanism. More specifically on that a Bitcoin peer stores addresses received from other peers and on that his database can be queried.

As was described in section II-A3 whenever a peer receives an unsolicited ADDR message, it stores the addresses from this message in his local database. The attacker can use this fact as follows. When a client connects to an attacker's peer, the peer sends him a unique combination of possibly fake addresses (address cookie) or fingerprint (we will use these two terms interchangeably below). Unique non-existent peer addresses work best, however a more sophisticated and more stealthy adversary may use existing Bitcoin peer addresses as well (exploiting the combinatorics of the coupon collector problem). The client stores these addresses and the next time he connects to (another) malicious peer, the peer queries his address database. If the fingerprint addresses are present in the set of retrieved addresses, the attacker identifies the user.

Consider a user $C$ and a set of Bitcoin servers $E_{1}, \ldots, E_{k}$ controlled by an attacker. Assume that one of the attacker's servers $E_{l}$ is among the user's entry nodes. The attacker executes the following steps:

1) Send a number of GETADDR messages to the user. The user should reply with ADDR messages.

2) Check the received from the client addresses if they already contain a fingerprint. If the user already has a fingerprint, stop. Otherwise go to the next step.

3) Generate a unique combination of $N$ fake addresses $F P$ and send them in an ADDR message to the client. The ADDR message should contain at least 11 addresses so that it is not forwarded by the client. If $N$ is less than 11, pad the message with $11-N$ legitimate ${ }^{14}$ addresses.

4) If the user connects to the Bitcoin network directly (i.e. without Tor), store the correspondence between the client's IP address and his fingerprint as a tuple $\left(F P, I P_{C}\right)$. If the user connects through Tor save him as $(F P$, NIL $)$.

There is a detail of the Bitcoin protocol which an attacker should take into account. As was described in subsection IIA1, when a client connects to the Bitcoin network over Tor, he will accept and store in his database OnionCat addresses only (thus ignoring IPv4 addresses). It means that in case of Tor, the fingerprint generated by the attacker should consist of OnionCat addresses only. On the other hand when a client connects to the network directly, he will ignore non-IPv4/IPv6 addresses. Hence an attacker should generate a fingerprint consisting of IPv4 addresses only. This results in that an attacker needs to store 2 different types of cookies: OnionCat and IPv4. At the same time, a client does not limit the types of addresses he sends as a reply to a GETADDR message. This means that once a cookie was set it can be queried both over Tor and directly.

\section{A. Stability of a Cookie}

According to the Bitcoin core [10] source code, at the startup when a client establishes outgoing connections he sends GETADDR messages, and gets back a set of addresses (typically 2,500, the maximum possible number per GETADDR request). Given 8 outgoing connection, the client will receive up to 20,000 non-unique addresses. These addresses can potentially overwrite the address cookie previously set by an attacker. Below we will try to estimate how this affects the stability of the cookie. Assume that an attacker managed to set an address cookie on a user's computer and disconnected (e.g. the client ended the session). The client then establishes a new session sometime later.

First note that if the user reconnects to Bitcoin over Tor and if the attacker has mounted the attack from section III, he

\footnotetext{
${ }^{14}$ By legitimate we mean that there are some Bitcoin servers running at these addresses.
} 
controls all user's traffic and the cookie is preserved. Let us now describe what happens if the client decides to connect to the Bitcoin network directly.

When a client receives an address $I P_{i n}$ he first checks if it is already contained in his database. If yes, he does nothing (thus the cookie is not damaged). In case it is a new IP address the client executes the following procedure. He computes the bucket number (see section II-A4) based on the peer which sent the address and the address itself. If this bucket contains a "terrible" 15 address $I P_{\text {terrible }}$, it is replaced by $I P_{i n}$. Otherwise 4 random addresses are chosen from the bucket and the one with the oldest timestamp is replaced by $I P_{\text {in }}$.

In other words, in order for the incoming address $I P_{\text {in }}$ to replace a cookie address $I P_{\text {cookie }}{ }^{16}$ the following conditions should hold:

1) $I P_{\text {in }}$ should not be in the user's database;

2) $I P_{\text {in }}$ should belong to the same bucket $B$ as $I P_{\text {cookie }}$ and there should be no "terrible" addresses in $B$;

3) IP $P_{\text {cookie }}$ should be among the four randomly chosen addresses, and its timestamp should be the oldest.

These conditions as we will see below make the attacker's cookie quite stable for many hours (this also depends on the number of user sessions since at each startup the address database is refreshed).

In order to estimate the probability that a cookie address set by the attacker is preserved we conducted the following experiment. In November 2014 we queried running Bitcoin servers by sending them GETADDR messages. We received 4,941,815 address-timestamp pairs. Only 303,049 of the addresses were unique. This can be interpreted as that only about $6 \%$ of the addresses received by a client will not be already contained in his database (if the client re-connects immediately).

As the second step, we looked at the timestamp distribution of the non-unique address set. This distribution can serve as approximation of the distribution of address timestamps of a client's database. The results are shown in Table I: $89 \%$ of addresses had a timestamp more than 3 hours in the past. Taking into account conditions stated above, it almost guarantees that the attacker's cookie will not be damaged within the first 3 hours. For $45 \%$ of addresses the timestamp was older than 10 hours (which is the duration of a working day); $9 \%$ of addresses were older than 1 week.

The results above could be summarized as follows: (1) there is a high chance that an address received by a client will already be contained in his database, which keeps the cookie intact; (2) if a cookie IP address is among the 4 nominees for erasing, it is likely that its timestamp will be fresher than that of at least one of other nominees (and thus will not be erased).

Finally we conducted the following experiment. We set a cookie consisting of $100 \mathrm{IPv} 4$ addresses and monitored how stable this cookie was across different sessions. Table II shows

\footnotetext{
${ }^{15} \mathrm{An}$ address is called terrible if any of the following holds: 1) its timestamp is 1 month old or more than 10 minutes in the future; 2) 3 consecutive connections to this address failed.

${ }^{16} \mathrm{~A}$ cookies consists of several IP address, but in order to make the explanation simpler, we use just one address here.
}

$\begin{array}{ccc}168(1 \text { week }) & 9 \% \\ \text { TABLE I. COMPLEMENTARY CUMULATIVE DISTRIBUTION FUNCTION }\end{array}$

\begin{tabular}{|c|c|}
\hline Address age, hours & 1-CDF \\
\hline \hline 3 & $89 \%$ \\
\hline 5 & $77 \%$ \\
\hline 10 & $45 \%$ \\
\hline 15 & $28 \%$ \\
\hline 24 & $19 \%$ \\
\hline 36 & $15 \%$ \\
\hline 48 & $13 \%$ \\
\hline 72 (3 days) & $12 \%$ \\
\hline 168 (1 week) & $9 \%$ \\
\hline
\end{tabular}

FOR ADDRESSES TIMESTAMPS

the decay rate of the number of cookie addresses over time and sessions. Note that by session we mean that the client switches off Bitcoin software and switches it on again, which forces him to make 8 new outgoing connections and retrieve up to 20,000 addresses.

\begin{tabular}{|c|c|c|}
\hline Session number & Time since start, hours & Remaining addresses \\
\hline \hline 1 & 0 & 100 \\
\hline 2 & 0.5 & 100 \\
\hline 3 & 1 & 100 \\
\hline 4 & 1.5 & 100 \\
\hline 5 & 2 & 100 \\
\hline 6 & 2.5 & 100 \\
\hline 7 & 3 & 98 \\
\hline 8 & 3.5 & 92 \\
\hline 9 & 5.5 & 50 \\
\hline 10 & 8 & 36 \\
\hline
\end{tabular}

TABLE II. ADDRESS COOKIE DECAY RATE (EXAMPLE)

The experiment shows that even after 10 sessions (i.e. after reception of about 200,000 non-unique IP addresses) and 8 hours, one third of the fingerprint remained in the user's database (thus it will be possible to identify the client). Note that sessions 9 and 10 took 2 and 2.5 hours. On the average an attacker will need about 90 peers (given that at the time of writing there are about 7,000 Bitcoin servers) to become one of the client's entry nodes during any of these 10 sessions and update the fingerprint. Running this number of peers will cost the attacker less than 650 USD per month (see section VII).

In another experiment we checked that in the case of two sessions with 10 hours between sessions, our client kept $76 \%$ of the initial fingerprint addresses, and in the case of 24 hours between two sessions $55 \%$ of the initial fingerprint were kept (which again allows the user identification). In order to carry out the experiments from this section we built our own rudimentary Bitcoin server which is able to connect/accept connections to/from Bitcoin peers and is capable of sending/receiving different Bitcoin messages on demand. We used this server as a malicious Bitcoin server which sets new address cookies and checks previously set cookies. In order to simulate a user we used the official Bitcoin core software (developed by the Bitcoin project) [10]. The attack from this section was experimentally verified by tracking our own clients in the real Bitcoin and Tor networks. 


\section{B. Cookie extraction}

The remaining question is how many GETADDR messages an attacker needs to send to the client to learn that the database of this client contains a cookie. According to [2], section 9.2 it can be up to 80 messages to retrieve the full collection of client's addresses. However in practice we will not need to collect all the addresses in a fingerprint, which significantly reduces the number of requests. About eight GETADDR messages would be sufficient to retrieve about $90 \%$ of the cookie addresses. This shows that the cookie can be checked without raising suspicion.

\section{Attack vectors}

Deanonymization of Bitcoin over Tor users. Consider the following case. A client uses the same computer for sending both benign Bitcoin transactions and sensitive transactions. For benign transactions the user connects to Bitcoin directly, but for sensitive transactions he forwards his traffic through a chain of Tor relays or VPNs. If an attacker implements the attack described in section III, all client's sensitive transactions with high probability will go through attacker's controlled nodes which will allow her to fingerprint the user and record his transactions.

When the client later connects to the Bitcoin network directly to send benign transactions, he will with some probability choose an entry node controlled by the attacker (in section $\mathrm{V}$ we show how to increase this probability). Once it happens, the attacker can query the client for the fingerprint and thus correlate his sensitive transactions with his IP address. Note that even if the attacker is not implementing the complete manin-the-middle attack on Tor, but just injects Sybil peers and Sybil hidden services she will be able to link many sensitive transactions to the real IP addresses of users.

Linking different Tor sessions. In the case, when a client uses a separate computer (or Bitcoin data folder ${ }^{17}$ ) to connect to Bitcoin through Tor, the attacker will not be able to learn his IP address. However, the attacker will still be able to link different transactions of the same user (remember that if a client sends a transaction through Tor the attacker can be certain that it was generated by this client). This can be done even across different sessions (computer restarts). This will in turn allow the attacker to correlate different Bitcoin addresses completely unrelated via transaction graph analysis.

Domino Effect. Tor multiplexes different streams of the same user over the same circuits. This means that if the source of one stream in the circuit is revealed by the fingerprinting attack, all other streams will also be deanonymized. Specifically, it is likely that a user who sends a sensitive Bitcoin transaction through Tor, will also browse a Darkweb site. Similar result was also noted in [16] but in relation to Bittorrent over Tor privacy issues. To prevent this it is recommended to enable option IsolateSOCKSAuth when running Tor (this will prevent sharing circuits with streams for which different SOCKS authentication was provided).

\footnotetext{
${ }^{17} \mathrm{~A}$ Bitcoin data folder is a directory where Bitcoin clients store their wallets and dump IP address databases between restarts.
}

\section{LOW-RESOURCE SYBIL ATTACKS ON BITCOIN}

In the previous section, we mentioned that a client needs to connect directly to one of the attacker's nodes in order to reveal his IP address so that an attacker can deanonymize his previous transactions done over Tor. Bitcoin as a peer-to-peer network is vulnerable to Sybil attacks and just operating many Bitcoin servers means that a client will sooner or later choose an entry node controlled by the attacker (i.e. in some number of sessions). However running too many servers can be costly (see section VII for attack cost estimation). Fortunately for the attacker there are a couple of ways to prevent Bitcoin clients from using non-attacker's Bitcoin servers (and choose an attacker's one instead).

\section{A. Exhausting connections limit}

As described in section II, by default a Bitcoin server accepts up to 117 connections. Once this limit is reached all new incoming connections are dropped. At the same time a Bitcoin server neither checks if some of these connections come from the same IP address ${ }^{18}$, nor forces clients to provide proof-ofwork. As a result a low-resource attacker can establish many connections to all but his Bitcoin servers ${ }^{19}$ and occupy all free connection slots. If a client connects directly to a Bitcoin server connection slots of which are occupied, the connection will be dropped immediately, thus the client will soon end up connecting to a malicious peer. This straightforward attack has been known in the Bitcoin community.

\section{B. Port poisoning attack}

A less effective but much stealthier new attack exploits the following fact. Peer addresses are of the following form (IP,PORT). However when a client decides if to add a received address to the database, he does not take the port number into account. For example assume a client receives an address $\left(I P_{0}, P O R T_{1}\right)$ and there is already an entry in the client's database $\left(I P_{0}, P O R T_{0}\right)$. In such case the client will keep $\left(I P_{0}, P O R T_{0}\right)$ and will not store $\left(I P_{0}, P O R T_{1}\right)$.

The attacker can use this fact to flood with clients with addresses of legitimate Bitcoin servers but wrong port numbers. If the attacker is the first to send such addresses, the client will not be able to connect to legitimate nodes.

\section{ESTIMATING CLIENT'S DELAYS}

The steps described in section III imply that once a client decides to use Bitcoin network over Tor, he will only be able to do this by choosing either one of the attacker's Exit nodes or one of the attacker's Bitcoin peers. However for the attack to be practical a user should not experience significant increases in connection delays. Otherwise the user will just give up connecting and decide that Tor-Bitcoin bundle is malfunctioning. In this section, we estimate the number of Bitcoin peers and the amount of bandwidth of Tor Exit relays which the attacker needs to inject, so that the attack does not degrade the user's experience.

\footnotetext{
${ }^{18}$ One explanation is that if clients are behind the same NAT they will share the same IP address.

${ }^{19}$ The list of all running Bitcoin servers can be obtained from e.g. [6].
} 
Once the attacker completes the steps described in section III, for each user connecting to the Bitcoin network through Tor there are several possibilities (see Fig. 1).

1) The user chooses one of the attacker's Bitcoin peers. The attacker does nothing in this case: the attacker automatically gains control over the information forwarded to the user.

2) The user chooses one of the attacker's Exit nodes. The attacker can use the fact that Bitcoin connections are not encrypted and not authenticated and redirect the client's request to Bitcoin peers under her control.

3) The user chooses a non-attacker's Exit relay and a running non-attacker's Bitcoin peer. In this case, due to the ban the user's connections will be rejected. And the user will try to connect to a different Bitcoin peer.

4) The user chooses a non-attacker's Exit relay and a non-attacker's Bitcoin peer which went offline ${ }^{20}$. In this case the Bitcoin client will wait until the connection times-out which can be up to two minutes (see section II-B1). This delay on the surface looks like taking prohibitively long time. However since during these two minutes Tor rebuilds new circuits every 10-15 seconds, trying new Exits at random, it actually makes the attacker's life easier. It increases the chances that malicious Exit relay will be chosen.

\section{B bitcoin}

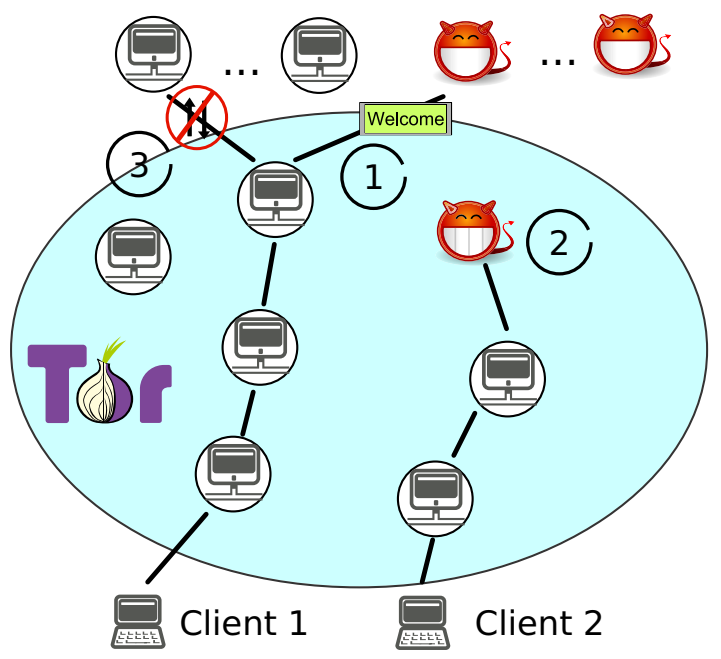

Fig. 1. Client's state after the main steps of the attack

\section{A. Handling unreachable Bitcoin peers}

Before estimating the delays we consider case 4 in more detail. Our experiments show that for a Bitcoin client which was already used several times prior to the connection over Tor, the address database contains $10,000-15,000$ addresses and the fraction of unreachable Bitcoin peers among them is between $2 / 3$ and $3 / 4$. Abundance of unreachable addresses means that case 4 is the most frequent scenario for the client.

\footnotetext{
${ }^{20}$ Or never really existed: Bitcoin allows storing fake addresses in client addresses database.
}

Consider a client which chose an unreachable Bitcoin server and a non-attacker's Exit node.

The Exit relay can send either: ${ }^{21}$

1) An END cell with a "timeout" error code. In case of a "timeout" message, Tor sends a "TTL expired" SOCKS error message to the Bitcoin application which then tries another Bitcoin peer.

2) An END cell with "resolve failed" error code ${ }^{22}$. In case of "resolve failed" message, Tor drops the current circuit and tries to connect to the unreachable Bitcoin peer through a different Exit node. After 3 failed resolves, Tor gives up and sends a "Host unreachable" SOCKS error code, which also results in Bitcoin trying a different peer.

3) The third and the most common option is that the exit relay will not send any cell at all during 10-15 seconds. As was described in the Background section that in case the Exit node does not send any reply within 10 or 15 seconds (depending on the number of failed tries) along the circuit attached to the stream, Tor drops the current circuit and attaches the stream to another circuit (or to a newly built one if no suitable circuits exist). In case Tor cannot establish connections during 125 seconds, it gives up and notifies Bitcoin client by sending a "General failure" SOCKS error message. Bitcoin client then tries another peer.

\section{B. Estimating delays}

The facts that a) Tor tries several different circuits while connecting to unreachable peers and b) the fraction of unreachable peers in the client's database is very large, significantly increases the chances that a malicious Exit node is chosen. The attacker only needs this to happen once, since afterwards all connections to the other Bitcoin peers will be established through this Tor circuit; Bitcoin client will work even with one connection. On the other hand, unreachable nodes increase the delay before the user establishes its first connection. This delay depends on the number of attacker's Bitcoin peers and on how often the user chooses new circuits.

In order to estimate the latter, we carried out the following experiment. We were running a Bitcoin client over Tor and for each connection to an unreachable Bitcoin client we were measuring the duration of the attempt and the number of new circuits (and hence different Exit nodes). The cumulative distribution function of the amount of time a Bitcoin client spends trying to connect to an unreachable node is shown in Fig. 2. On the average a Bitcoin peer spends 39.6 seconds trying to connect to an unreachable peer and tries to establish a new circuit (and hence a different Exit node) every 8.6 seconds. This results in 4.6 circuits per unreachable peer on the average.

We now estimate how long it will take a user on the average to establish his first connection to the Bitcoin network. This delay obviously depends on the number of the attacker's Bitcoin peers and the amount of bandwidth of her Tor Exit relays. We adopt a simple discrete time absorbing Markov chain model with only three states (see Fig. 3):

\footnotetext{
${ }^{21}$ This is based on the Tor source code analysis and monitoring a running Tor instance.

${ }^{22}$ We observed this behaviour not only for hostnames but also for IP addresses.
} 


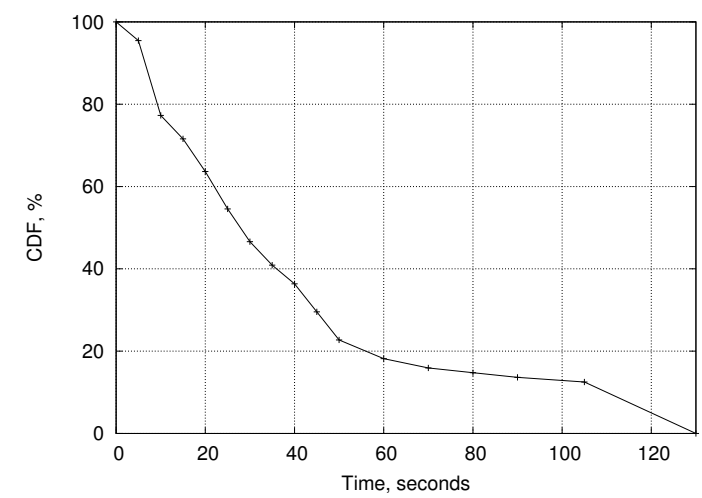

Fig. 2. Time spent connecting to an unreachable node

- State 1: the Bitcoin client tries to connect to an unreachable peer;

- State 2: the Bitcoin client tries to connect to a reachable Bitcoin peer banned by the attacker;

- State 3: the Bitcoin client tries to connect to an attacker's Bitcoin peer or chooses an attacker's Tor Exit node. State 3 is absorbing state, once it is reached, the user thinks that he connected to the Bitcoin network (while he is now controlled by the attacker).

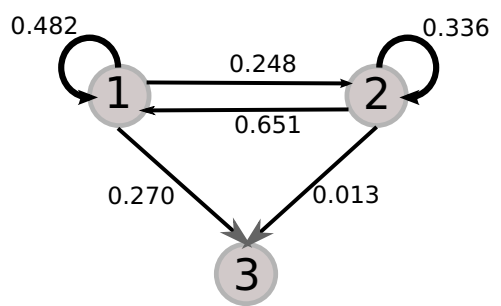

Fig. 3. Markov chain with probabilities for $400 \mathrm{~K}$ of Exit capacity and 100 malicious Bitcoin peers. The client spends about 0.5 seconds in State 2 and about 40 seconds in State 1

After composing the fundamental matrix for our Markov chain, we find the average number of steps in two non-absorbing states. Taking into account the average amount of time spent by the user in each of the states (we use our experimental data here), we find the average time before the absorbing state. We compute this time for different number of Bitcoin peers and Tor Exit relay bandwidth. The results are presented in Fig 4. We have taken a conservative estimate that the fraction of unreachable Bitcoin peers in the client's database is $2 / 3=$ $66 \%$, also the client spends only about 0.5 seconds in State 2 and about 40 seconds in State 1.

Fig. 4 shows that an attacker having 100,000 of consensus Exit bandwidth and 1000 Bitcoin peers is able to carry out the attack while keeping the average delay below 5 minutes. For example an attacker controlling a small botnet can afford that many peers (she will need 1000 peers with public IPs or supporting UPnP protocol). An attacker having consensus weight of 400,000 and very few peers can decrease the average delay to about two minutes. Such a bandwidth is achievable by an economy level attacker as will be shown in section VII.

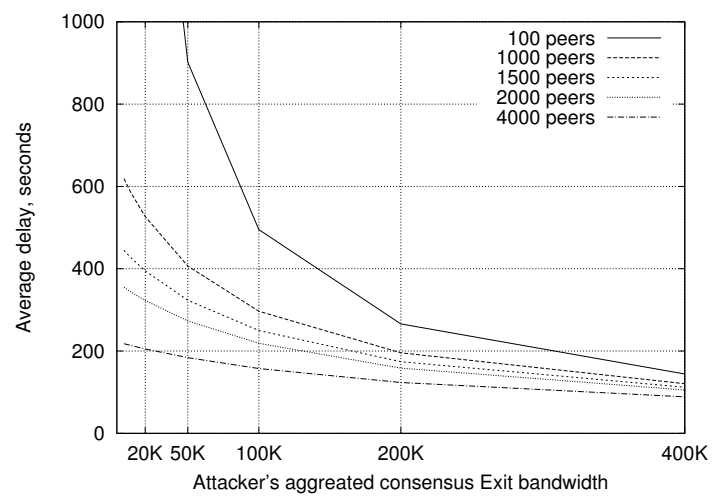

Fig. 4. Average time before the first connection

The line corresponding to 4000 attacker's Bitcoin peers in Fig. 4 is not as unrealistic as it may seem. Recall (see Section II-A4) that each Bitcoin peer address can go to up to 4 "new" buckets at client's side. This can be used by a persistent attacker to increase the choice probability for her peers by a factor 4 (in the best case) which means an attacker can have significantly less than 4000 peers.

\section{Clients with empty addresses cache}

As was pointed in Section II-A3, all IPv4 and IPv6 addresses received from DNS-oneshots are dropped by a Bitcoin client if Tor is used. If the addresses database of a client is empty and all the seed nodes are banned, the client can connect to hidden services only. This is a limitation of our approach.

\section{ATTACK COSTS}

\section{A. Tor Exit nodes}

During July 2014 we were running a non-Exit Tor relay for 30 USD per month. We set the bandwidth limit of the relay to $5 \mathrm{MB} / \mathrm{s}$ which resulted in traffic of less than $15 \mathrm{~GB}$ per hour. The consensus bandwidth of this relay fluctuated between 5,000 and 10,000 units ${ }^{23}$. While the total weighted consensus bandwidth of all exit nodes was about 7 million units, the weighted consensus bandwidth of relays allowing exiting at port 8333 was about 5.7 million units. Assuming that we could achieve 5,000 - 10,000 units in the consensus for an Exit node this gives the probability of $0.08 \%-0.17 \%$ for our relay to be chosen for Exit position by a user. Given that $10 \mathrm{~TB}$ of traffic is included into the server's price and one has to pay 2 EUR per additional $1 \mathrm{~TB}$, it would cost an attacker 360 USD to have $180 \mathrm{~TB}$ of traffic per month. The corresponding speed is $69 \mathrm{MB} / \mathrm{s}(69,000$ consensus bandwidth units). By running 6 such relays the attacker can achieve $400 \mathrm{~K}$ of bandwidth weight in total for the price below 2500 USD (2160 USD for the traffic and 240 for renting fast servers).

Thus having a consensus weight close to 400,000 is possible for an economy-level attacker. The attacker can also decide to play unfair and mount a bandwidth cheating attack which would allow her to have a high consensus weight while keeping the budget of the attack even lower [22]. This is especially

\footnotetext{
${ }^{23} \mathrm{~A}$ unit roughly corresponds to $1 \mathrm{~KB} / \mathrm{s}$ of traffic.
} 
possible since Bitcoin traffic by itself is rather lightweight and high bandwidth would be needed only in order to drive Tor path selection algorithm towards attacker's nodes.

\section{B. Bitcoin peers}

The attack described in sections III and IV suggests the attacker injects a number Bitcoin peers; at the same time Bitcoin network allows only one peer per IP address. Thus the attacker is interested in getting as many IP addresses as possible. Currently there are several options. The cheapest option would be to rent IP addresses on per hour basis. The market price for an IP address is 1 cent per hour [21]. This results in 7200 USD per 1000 IP's per month. From these computations it is clear that an attacker would do better by investing in Exit bandwidth rather than running Bitcoin peers (unless she controls a small botnet), and the only limitation for her would be not to become too noticeable. An attacker that has $400 \mathrm{~K}$ ( $7 \%$ for port 8333 ) of Tor Exit capacity would cost about 2500 USD.

\section{COUNTERMEASURES}

These attacks are very effective due to a feature of Bitcoin which allows an easy ban of Tor Exit nodes from arbitrary Bitcoin peers and due to easy user fingerprinting with the "address cookies". One possible countermeasure against Torban could be to relax the reputation-based DoS protection. For example each Bitcoin peer could have a random variable, which would decide whether to turn ON or OFF the DoS protection mechanism with probability $1 / 2$. As a result the attacker might be able to DoS at most half of the network, but on the other hand he will not be able to ban any relays or VPNs from all the Bitcoin peers.

An obvious countermeasure would be to encrypt and authenticate Bitcoin traffic. This would prevent even opportunistic man-in-the-middle attacks (i.e. even if the user is unlucky to choose a malicious Exit relay). Another possible countermeasure is to run a set of "Tor-aware" Bitcoin peers which would regularly download Tor consensus and make sure that Bitcoin DoS countermeasures are not applied to servers from the Tor consensus. K. Atlas [1] implemented a similar countermeasure (which maintains historical record of Tor exit nodes used to connect to the Bitcoin network.)

Finally, Bitcoin developers can maintain and distribute a safe and stable list of onion addresses. Users which would like to stay anonymous should choose at least one address from this list. There currently exists a short and not up-to-date list of Bitcoin fallback onion addresses [12]. Another advice for a user would be to run two Bitcoin nodes, one over Tor and one without, and compare their blockchains and unconfirmed transactions. This would prevent from creation of virtual reality for Tor-only users.

With regards to the fingerprinting attack several countermeasures are possible. First, Bitcoin peers can request performing proof-of-work computation for each sent GETADDR message, so that it becomes computationally expensive for an attacker to query each client. Second, according to the Bitcoin core source, the only time when a client sends a GETADDR message is when he establishes an outbound connection. Thus ignoring GETTADDR requests on outbound connections will not change the usual operation of Bitcoin networking protocol and will prevent the attacker from requesting the fingerprint ${ }^{24}$. Finally an immediate countermeasure would be to remove the cached address database file before each session and to use only trusted hidden-services.

\section{REFERENCES}

[1] K. Atlas, "Historical record of Tor exit nodes used to connect to the Bitcoin," http://www.openbitcoinprivacyproject.org/torban/www/, 2014.

[2] A. Biryukov, D. Khovratovich, and I. Pustogarov, "Deanonymisation of clients in Bitcoin P2P network," CoRR, vol. abs/1405.7418, 2014.

[3] A. Biryukov, I. Pustogarov, and R.-P. Weinmann, "Trawling for tor hidden services: Detection, measurement, deanonymization," in Proceedings of IEEE Symposium on Security and Privacy (SP'13). IEEE Computer Society, 2013.

[4] Bitcoin Wiki, https://en.bitcoin.it/wiki/, 2014.

[5] BitcoinJ - a Java implementation of the Bitcoin protocol, https://github.com/bitcoinj/bitcoinj, 2014.

[6] Bitnodes, https://getaddr.bitnodes.io/, 2014

[7] D. Bradbury, "Could eroding net neutrality hurt bitcoin?" CoinDesk, 2014, http://www.coindesk.com/eroding-net-neutrality-hurt-bitcoin/.

[8] A. Cuthbertson, "Bitcoin now accepted by 100,000 merchants worldwide," International Business Times (IBTimes), 2014, http://www.ibtimes.co.uk/bitcoin-now-accepted-by-100000-merchantsworldwide-1486613.

[9] Double-spending, https://en.bitcoin.it/wiki/Double-spending, 2014.

[10] Download Bitcoin Core, https://bitcoin.org/en/download, 2014.

[11] Electrum Bitcoin client, https://electrum.org/, 2011.

[12] Fallback Nodes, https://en.bitcoin.it/wiki/Fallback_Nodes, 2014.

[13] M. Hearn, "Exiting only port 8333: Motivation for Bitcoinj+Tor client," https://lists.torproject.org/pipermail/tor-relays/2014March/004145.html, 2014.

[14] D. Kaminsky, "Black ops of TCP/IP," Black Hat USA, 2011, http://www.slideshare.net/dakami/black-ops-of-tcpip-2011-black-hatusa-2011.

[15] P. Koshy, D. Koshy, and P. McDaniel, "An analysis of anonymity in bitcoin using P2P network traffic," in Proceedings of Financial Cryptography and Data Security (FC'14). Springer, 2014.

[16] P. Manils, C. Abdelberi, S. L. Blond, M. A. Kâafar, C. Castelluccia, A. Legout, and W. Dabbous, "Compromising Tor anonymity exploiting P2P information leakage," CoRR, vol. abs/1004.1461, 2010.

[17] S. Meiklejohn, M. Pomarole, G. Jordan, K. Levchenko, D. McCoy, G. M. Voelker, and S. Savage, "A fistful of Bitcoins: Characterizing payments among men with no names," in Proceedings of the 2013 Conference on Internet Measurement Conference (IMC'13). ACM, 2013.

[18] S. Nakamoto, "Bitcoin: A peer-to-peer electronic cash system," 2009, http://www. bitcoin.org/bitcoin.pdf.

[19] OnionCat: An Anonymous VPN-Adapter, https://www.onioncat.org/about-onioncat/, 2014.

[20] D. Ron and A. Shamir, "Quantitative analysis of the full Bitcoin transaction graph," in Proceedings of Financial Cryptography and Data Security (FC'13). Springer, 2013.

[21] Terremark vCloud Express, http://vcloudexpress.terremark.com/pricing.aspx, 2014.

[22] F. Thill, "Hidden Service Tracking Detection and Bandwidth Cheating in Tor Anonymity Network. Master Thesis," 2014, https://www.cryptolux.org/images/b/bc/.

[23] Tor FAQ, https://www.torproject.org/docs/faq.html.en\#TBBFlash, 2014.

[24] Tor security advisory: "relay early" traffic confirmation attack, https://blog.torproject.org/blog/tor-security-advisory-relay-early-trafficconfirmation-attack, 2014.

[25] Torsocks: use socks-friendly applications with Tor, https://code.google.com/p/torsocks/, 2014.

\footnotetext{
${ }^{24}$ We implemented this countermeasure and submitted the corresponding
} 


\section{APPENDIX A \\ List OF REACHABLE BITCOIN ONIONS}

In this Appendix we list 39 Bitcoin onion addresses which we found to be reachable in August 2014 and 46 onion addresses reachable in November 2014. In order to get this list we queried reachable for the time of the experiments Bitcoin peers by sending four GETADDR messages to each peer. A Bitcoin peer can reply to such message by sending back $23 \%$ of its addresses database but not more than 2500 addresses. A peer can store 20,480 addresses at most which means that sending 4 GETADDR messages is not enough to extract the complete peer's database. However we expect that there is a big overlap between the databases of different peers. Some of the discovered reachable onion addresses begin or end with meaningful text like: BTCNET, BITCOIN and belong to Bitcoin developers, pools or services.

\begin{tabular}{|l|l|}
\hline 2fvnnvj2hiljjwck.onion:8333 & it2pj4f7657g3rhi.onion:8333 \\
\hline 2zdgmicx7obtivug.onion:8333 & jq57qrkvvyi4a3o2.onion:8333 \\
\hline 3crtkleibhn6qak4.onion:14135 & kjy2eqzk4zwi5zd3.onion:8333 \\
\hline 3lxko7l4245bxhex.onion:8333 & mtzcz5knzjmuclnx.onion:8333 \\
\hline 4crhf372poejlc44.onion:8333 & nns4r54x3lfbrkq5.onion:8333 \\
\hline 5ghqw4wj6hpgfvdg.onion:8333 & nzsicg2ksmsrxwyz.onion:8333 \\
\hline 5k4vwyy5stro33fb.onion:8333 & pqosrh6wfaucet32.onion:8333 \\
\hline 6fp3i7f2pbie7w7t.onion:8333 & pt2awtcs2ulm75ig.onion:8333 \\
\hline 7iyfdkr72hgtdjoh.onion:8333 & pxl7ytsd2aiydadi.onion:8333 \\
\hline b6fr7dlbu2kpiysf.onion:8333 & qsxhkpvbmt6akrov.onion:8333 \\
\hline bitcoin625tzsusi.onion:8333 & syix2554lvyjluzw.onion:8333 \\
\hline bitcoinostk4e4re.onion:8333 & t2vapymuu6z55s4d.onion:8333 \\
\hline btcdatxubbzaw4tj.onion:8333 & td7tgof3imei3fm6.onion:8333 \\
\hline btcnet3utgzyz2bf.onion:8333 & tfu4kqfhsw5slqp2.onion:8333 \\
\hline czsbwh4pq4mh3izl.onion:8333 & thfsmmn2jbitcoin.onion:8333 \\
\hline dqretelgl3kjtzei.onion:8333 & xdnigz4qn5dbbw2t.onion:8333 \\
\hline e3tn727fywnioxrc.onion:8333 & xij5qyrbosw2pzjm.onion:8333 \\
\hline evolynhit7shzeet.onion:8333 & zqq6yxxxb7or36br.onion:8333 \\
\hline gb5ypqt63du3wfhn.onion:8333 & zy3kdqowmrb7xm7h.onion:8333 \\
\hline hkxy4jpeniuwouiv.onion:8333 & \\
\hline
\end{tabular}

TABLE III. Bitcoin ONions, OnLine In August 2014

\begin{tabular}{|l|l|}
\hline 2xylerfjgat6kf3s.onion:8333 & h2vlpudzphzqxutd.onion:8333 \\
\hline 2zdgmicx7obtivug.onion:8333 & hkxy4jpeniuwouiv.onion:8333 \\
\hline 3ffk7iumtx3cegbi.onion:8333 & iksneq25weneygcj.onion:8333 \\
\hline 3lxko7l4245bxhex.onion:8333 & k22qrck6cetfj655.onion:8333 \\
\hline 4crhf372poejlc44.onion:8333 & kjy2eqzk4zwi5zd3.onion:8333 \\
\hline 5k4vwyy5stro33fb.onion:8333 & lazsruhzupsgpvwm.onion:8333 \\
\hline 6fizop6wctokuxyk.onion:8333 & lfmwsd65ltykrp74.onion:8333 \\
\hline 6fp3i7f2pbie7w7t.onion:8333 & luruc27g24y7ewwi.onion:8333 \\
\hline 7g7j54btiaxhtsiy.onion:8333 & pqosrh6wfaucet32.onion:8333 \\
\hline 7pkm6urc5hlgwlyc.onion:8333 & pt2awtcs2ulm75ig.onion:8333 \\
\hline b2ykuvob44fn36wo.onion:8333 & pxl7ytsd2aiydadi.onion:8333 \\
\hline b6fr7dlbu2kpiysf.onion:8333 & qsntokcdbwzmb2i5.onion:8333 \\
\hline bitcoinostk4e4re.onion:8333 & sbow7bnje2f4gcvt.onion:8333 \\
\hline bk5ejfe56xakvtkk.onion:8333 & td7tgof3imei3fm6.onion:8333 \\
\hline btc4xysqsf3mmab4.onion:8333 & tfu4kqfhsw5slqp2.onion:8333 \\
\hline btcnet3utgzyz2bf.onion:8333 & thfsmmn2jbitcoin.onion:8333 \\
\hline by4ec3pkia7s7wy2.onion:8333 & ukronionufi6qhtl.onion:8333 \\
\hline dioq2yg3l5ptgpge.onion:8333 & vqpye2k5rcqvj5mq.onion:8333 \\
\hline dqretelgl3kjtzei.onion:8333 & wc5nztpe26jrjmoo.onion:8333 \\
\hline drp4pvejybx2ejdr.onion:8333 & xudkoztdfrsuyyou.onion:8333 \\
\hline e3tn727fywnioxrc.onion:8333 & z3isvv4llrmv57lh.onion:8333 \\
\hline evolynhit7shzeet.onion:8333 & zc6fabqhrjwdle3b.onion:8333 \\
\hline gb5ypqt63du3wfhn.onion:8333 & zy3kdqowmrb7xm7h.onion:8333 \\
\hline
\end{tabular}

TABLE IV. BITCOIN ONIONS, ONLINE IN NOVEMBER 2014

\section{APPENDIX B}

\section{DOUBLE-SPENDING}

In this section we describe three techniques which an attacker can use to try to carry out a double spend attack.
These techniques are known to the bitcoin community and described in e.g. [9].

\section{A. Race attack (0-confirmation attack)}

The first method assumes that a merchant accepts a payment immediately upon receipt of the corresponding transaction (i.e. he does not wait until this transactions is included into a block) and gives away the product to the client. In such case a malicious client can communicate one transaction (with the payment) to the merchant and a different transaction which spends the same inputs to the rest of the network. This will result in that only the second transaction will be included by miners into the new block, and thus accepted by the network while the first transaction (i.e. the one which the merchant received) will finally be rejected by the network.

The attack is successful only if the attacker manages to deliver the second transaction to miners before the first transaction is broadcasted to the network by the merchant. In case the attacker controls merchant's connections to the network (as in Bitcoin over Tor attack described in this paper), it is much easier for the attacker to succeed. The common use-case for this attack would be when it is unlikely that a client would wait for 10-minutes confirmation before taking his purchase and go.

\section{B. 1-block confirmation attack ${ }^{25}$}

The target of the second attack is a service which allows one to make a deposit (in Bitcoins) by sending it in the corresponding transaction and withdraw the deposited Bitcoins back as soon as the transaction is included into a block. The service should use different coins for the withdrawal than the ones that were just received for the deposit.

An attacker creates a transaction which makes a large deposit (e.g. 50 BTC) to the service and adds it to a block that she is currently mining. The transaction is kept private. When the attacker finds a valid block she does not broadcast it immediately, but instead waits until someone else mines another block. Once it happens, the attacker sends his block to the service. If the attacker's block arrives first, the service accepts the deposit transaction which will have one confirmation. At the same time the rest of the network accepts the other block ${ }^{26}$. The attacker immediately request a withdrawal, and the service generates a transaction sending the large amount of coins to the attacker. Since the rest of the network is working on a different blockchain fork, the attacker's deposit will soon be invalidated while the withdrawal will be considered valid.

The attack only succeeds if the attacker manages to deliver his block to the service first while the rest of the network (or more specifically the majority of miners) accepts a different block. The success is much more likely if the attacker controls the service's network connections.

\footnotetext{
${ }^{25}$ This attack was described by user Vector76 in a Bitcoin forum post.

${ }^{26}$ The attacker also double-spends the inputs from the deposit transaction. The network (since it did not receive the attacker's block) will accept it as valid and work to include it in the next block.
} 
C. Finney attack (block withholding)

The third attack similarly to the Race attack assumes that a merchant accept a payment upon receipt of the unconfirmed transaction. A malicious miner generates a transaction in which he sends bitcoins to himself and includes it to the block he is working on. The transaction is kept private.
When the block is found, the miner spends the same coins somewhere else and immediately after that releases the block. As the result, the network accepts the block and considers the transaction to the merchant as invalid. 